The Astronomical JournaL, 118:1034-1042, 1999 August

(C) 1999. The American Astronomical Society. All rights reserved. Printed in U.S.A.

\title{
NOVA SAGITTARII 19941 (V4332 SAGITTARII): THE DISCOVERY AND EVOLUTION OF AN UNUSUAL LUMINOUS RED VARIABLE STAR
}

PaUl Martini AND R. MARK WAGNeR ${ }^{1}$

Department of Astronomy, Ohio State University, 174 West 18th Avenue, Columbus, OH 43210; martini@astronomy.ohio-state.edu, rmw@as.arizona.edu AUSTIN TOMANEY

Department of Astronomy, P.O. Box 351580, University of Washington, Seattle, WA 98195

R. Michael Rich

Department of Physics and Astronomy, UCLA, 405 Hilgard Avenue, Los Angeles, CA 90095-1547

M. Della Valle

Dipartimento di Astronomia, Vicolo dell'Osservatorio, 5, I-35122 Padova, Italy

AND

Peter H. Hauschildt

Department of Physics and Astronomy and the Center for Simulational Physics, University of Georgia, Athens, GA 30602-2451

Received 1997 August 25; accepted 1999 May 3

\begin{abstract}
We report photometry and spectroscopy of the evolution of Nova Sagittarii 19941 (V4332 Sagittarii) during outburst. We compare the photometric and spectral evolution of this outburst with known classes of outbursts - including classical novae and outbursts occurring on symbiotic stars - and find this object does not conform to any known class of outburst. The closest match to the behavior of this unusual object is M31 RV, an extremely luminous and red variable object discovered in the bulge of M31 in 1988. However, the temporal behavior and maximum luminosity of the two events differ by several orders of magnitude, requiring substantial intrinsic variation if these two events are members the same type of outburst. Our model of the spectroscopic evolution of this outburst shows that the effective temperature cooled from 4400 to $2300 \mathrm{~K}$ over the 3 month span of our observations. In combination with line diagnostics in our later spectra, including [ [ $\left.\mathrm{O}_{\mathrm{I}}\right] \lambda 5577$ and the dramatic increase in the $\mathrm{H} \alpha-$ to- $\mathrm{H} \beta$ ratio, we infer the existence of a cool, dense $\left(N_{e} \sim 10^{8}-10^{9} \mathrm{~cm}^{-3}\right)$ envelope that is optically thick in the hydrogen Balmer recombination lines (case $\mathrm{C}$ ). We suggest that a nuclear event in a single star, in which a slow shock drove the photosphere outward, can power the observed luminosity evolution and the emission spectrum.
\end{abstract}

Key words: stars: evolution - stars: peculiar — stars: variables: other

\section{INTRODUCTION}

On 1994 February 24, M. Yamamoto discovered an apparent nova in Sagittarius, Nova Sagittarii 19941 (hereafter V4332 Sgr; Hayashi, Yamamoto, \& Hirosawa 1994). A confirmation spectrum obtained by R. Bertram with the Perkins telescope on 1994 March 4 indicated that this object was not a classical nova early in its outburst (Wagner 1994) since it lacked the spectral features characteristic of classical novae in outburst. Subsequent spectra also showed this object to undergo approximately a $1 \mathrm{mag}$ decline in flux and a change in spectral type from approximately M0 to M5 in only 5 days (Tomaney et al. 1994). Based upon its evolution toward later spectral type and the lack of any emission other than narrow Balmer lines, Tomaney et al. (1994) proposed that V4332 Sgr was of the same type as an extremely luminous red variable observed in the bulge of M31 (hereafter M31 RV) in 1988 (Rich et al. 1989; Mould et al. 1990; Sharov 1990, 1993; Tomaney \& Shafter 1992). M31 RV similarly exhibited only narrow Balmer emission, although this object evolved on a much longer timescale and attained a much brighter absolute magnitude than V4332 Sgr.

\footnotetext{
${ }^{1}$ Current Address: Steward Observatory, University of Arizona, Tucson, AZ 85721.
}

In this paper, we compile all available observations of V4332 Sgr during outburst and show that its spectroscopic and photometric evolution are inconsistent with any known class of outburst. In $\S 2$, we describe the discovery and identification of V4332 Sgr. In $\S \S 3$ and 4 we trace its photometric and spectroscopic evolution, while in $\S 5$ we discuss our model fits to our spectra and derive effective temperatures for the expanding shell as a function of time. In $\S 6$ we describe high-resolution spectroscopy of $\mathrm{H} \alpha$ at two epochs, while in $\S \S 7$ and 8 we compare V4332 Sgr with other known classes of outbursts and M31 RV. We briefly discuss the possible nature of the object and this outburst in $\S 9$, and we summarize our results in $\S 10$.

\section{DISCOVERY, IDENTIFICATION, AND ASTROMETRY}

V4332 Sgr was discovered by M. Yamamoto on an exposure with P-MAX 400 film on 1994 February 24.85 UT at a magnitude of 8.9 (Hayashi et al. 1994). The presence of the new object was confirmed visually by Hirosawa on February 25.85 at a magnitude of about 8.4. Further observations by Yamamoto on February 25.84 gave a magnitude estimate of 8.5, consistent with the results of Hirosawa (Hayashi et al. 1994). V4332 Sgr should not be confused with a second nova in Sagittarius (Nova Sagittarii 1994 2) discovered by Sakurai (Sato et al. 1994) on 1994 May 20.7.

In Figure 1, we show the location of V4332 Sgr based on a 10 s $R$-band CCD image obtained on 1994 March 22 


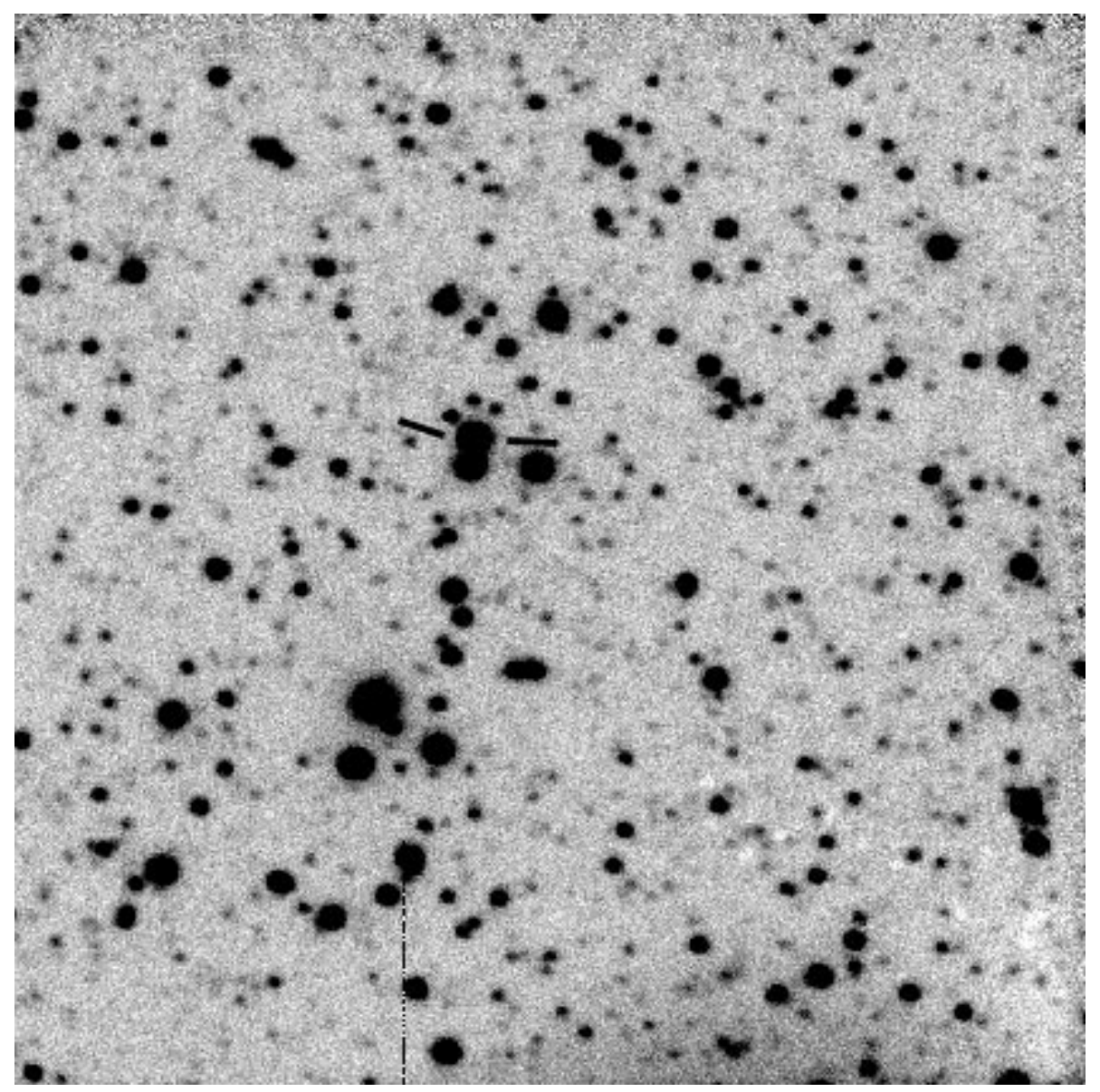

Fig. 1.-A $10 \mathrm{~s} R$-band exposure of V4332 Sgr obtained on 22 March 1994 at the $1.8 \mathrm{~m}$ Perkins telescope with the Imaging Fabry Perot Spectrograph in direct mode. The field is approximately $6^{\prime}$ on a side. North is up, and west is to the right.

using the Imaging Fabry-Perot Spectrometer (Pogge et al. 1995 ) in direct imaging mode and a TI $800 \times 800$ pixel CCD mounted on the Perkins telescope of the Ohio Wesleyan and Ohio State Universities at the Lowell Observatory. Figure 1 covers a region $6^{\prime} .0 \times 6.3$ at a scale of 0.5 pixel $^{-1}$. The seeing was approximately $2^{\prime \prime}$ FWHM.

Gilmore et al. (1994) obtained astrometry of V4332 Sgr by utilizing the Parallax and Proper Motion Catalog (PPM) based on two exposures obtained by A. C. Gilmore with the $0.15 \mathrm{~m} \mathrm{f} / 15$ astrograph at Mount John Observatory on March 2.62 UT. Kilmartin determined a mean FK5/J2000.0 position of $\alpha=18^{\mathrm{h}} 50^{\mathrm{m}} 36^{\mathrm{s}} .74, \delta=$ $-21^{\circ} 23^{\prime} 28^{\prime \prime} 8$ (Wagner et al. 1994). Skiff (1994) examined the Palomar Observatory Sky Survey (POSS) O and E prints and found a possible progenitor of the nova at this position with $R=16 \mathrm{mag}$ and $B=18 \mathrm{mag}$ at a mean position of $\alpha=18^{\mathrm{h}} 50^{\mathrm{m}} 36^{\mathrm{s}} .80, \delta=-21^{\circ} 23^{\prime} 29^{\prime \prime} .3(\mathrm{~J} 2000.0)$ (Wagner et al. 1994). With our estimated extinction of $A_{V}=1$ (see below), this is roughly consistent with a $\mathrm{K}$ star. This is also consistent with the initial spectral type of the outburst.

From the $R$-band CCD images we obtained on 1994 March 22, we derived an astrometric solution for 27 stars that were in common with our CCD image and the Digitized Sky Survey based upon a grid of 18 PPM stars in a $100^{\prime} \times 100^{\prime}$ field centered on V4332 Sgr. Using our plate solution, we obtained a position of $\alpha=18^{\mathrm{h}} 50^{\mathrm{m}} 36^{\mathrm{s}} .73, \delta=$ $-21^{\circ} 23^{\prime} 28^{\prime \prime} .98$ (J2000.0) with a formal uncertainty of 0 ".26 in both coordinates. Our position is consistent at the $1 \sigma$ level with the position derived by Kilmartin and with the position of the possible progenitor on the POSS print discovered by Skiff.

\section{LIGHT CURVE}

In Figure $2 a$, we plot the light curve of V4332 Sgr as compiled by the Variable Star Network, the AAVSO, and photometry reported in the IAU Circulars $(5943,5944$, and 5949). We show digital measurements obtained by photoelectric photometry or with CCDs as filled circles, while we denote visual magnitude estimates by open circles. Visual estimates were obtained and kindly communicated to us by A. Jones of the Variable Star Section of the Royal Astronomical Society of New Zealand using a $0.32 \mathrm{~m}$ telescope. These points are shown as open triangles. The photographic observations, primarily by Gilmore et al. (1994), largely precede the discovery observation and are shown as filled triangles. For convenience, we have included the results of published photoelectric photometry in Table 1.

The light curve is characterized by a slow rise in brightness to a relatively flat maximum. Maximum brightness appears to have been reached on JD 2,449,412 \pm 1 (1994 February 28) at a visual magnitude of $\sim 8.5$. This broad maximum was then followed by a relatively fast decay in which the time to decline by 2 and 3 magnitudes in $V$ was $t_{2}=8 \pm 1$ and $t_{3}=11 \pm 1$ days, respectively. In addition, the visual magnitude measurements suggest that the rate of decline slowed after JD 2,449,424 (1994 March 12). We per- 


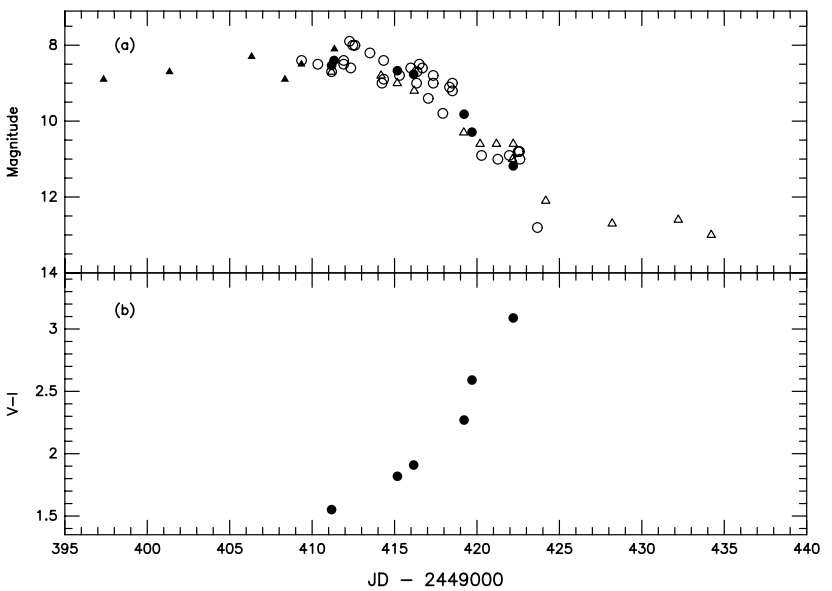

FIG. 2.-Light curve of V4332 Sgr during outburst: (a) Evolution of V4332 Sgr in the visible. In this figure, filled triangles correspond to photographic measurements, open triangles correspond to visual estimates, open circles represent visual magnitude estimates, and filled circles correspond to photoelectric photometry. (b) $V-I$ light curve over the same period of time, where the filled circles represent photoelectric photometry as in $(a)$. The $1 \sigma$ uncertainties in the photoelectric photometry are smaller than the size of the symbols. The maximum is roughly at JD 2,449,412, corresponding to 1994 February 28 . See $\S 3$ for further details.

formed a crude linear back-extrapolation of the premaximum light-curve data (Fig. 2a; filled triangles) and estimate that the object took $\sim 200$ days to reach maximum brightness, assuming a quiescent magnitude of $B \simeq 18 \mathrm{mag}$ based on Skiff's proposed progenitor. Archival plates examined by Wenzel (1994) indicate that V4332 Sgr was not visible on 561 Sonneberg sky patrol plates taken between 1926 and 1983 to a limiting photographic magnitude of 11-13.5. We note that this object was just becoming visible in the morning sky when it was discovered, and therefore more complex photometric behavior prior to its discovery cannot be excluded.

In Figure $2 b$, we show the $V-I$ color evolution taken from the photometry reported by Gilmore et al. (1994; IAU Circulars 5943, 5944, and 5949) starting from maximum light and extending for 11 additional days (see also Table 1). During this time, the slope of the continuum of V4332 Sgr as measured by the $V-I$ color became redder by $\sim 1.5$ mag, while the object faded by $2.5 \mathrm{mag}$ in $V$. This suggests that much of the steep decline in the visual magnitude was the result of a rapidly reddening continuum. This behavior is confirmed by the spectral evolution of V4332 Sgr discussed below.

\section{SPECTRAL EVOLUTION}

We followed the early spectral evolution of V4332 Sgr from 1994 March 4 to June 6 at a variety of telescopes (Table 2). In Figure 3, we present spectra of V4332 Sgr obtained at the Perkins Telescope on 1994 March 4.5 (Fig. $3 a$ ) and 9.5 UT (Fig. $3 b$ ) with the Boller and Chivens CCD spectrograph. We employed a 350 grooves $\mathrm{mm}^{-1}$ grating centered at $5100 \AA$ and a $2^{\prime \prime}$ wide slit to cover the spectral region from $4500-5700 \AA$ at a resolution of $5 \AA$. Upon comparing this spectrum with the Turnshek et al. (1985) atlas of cool stars and the spectra presented by Jacoby, Hunter, \& Christian (1984), we concluded that the March 4 spectrum (Fig. 3a) most closely resembles a K3-4 III-I star.

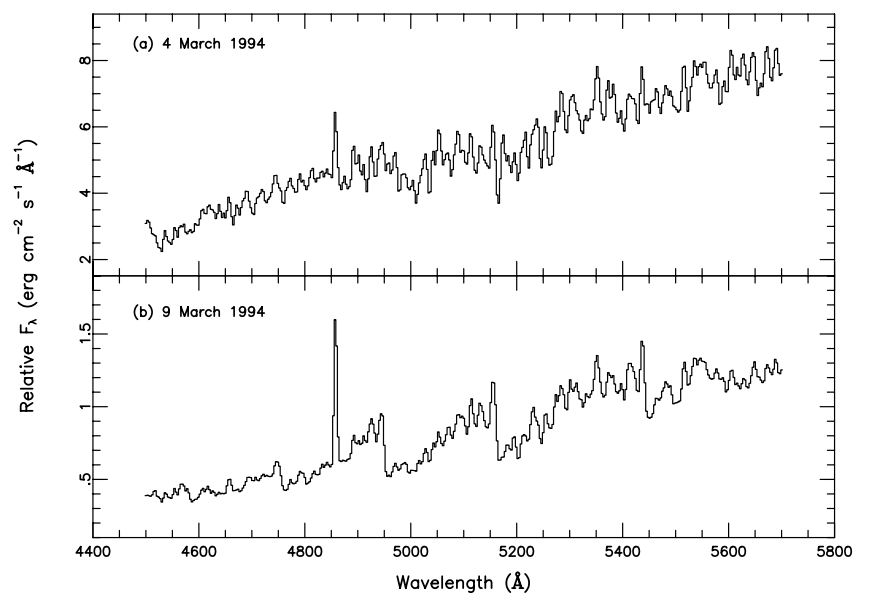

FIG. 3.-March 4 and 9 spectra obtained at the Perkins $1.8 \mathrm{~m}$ telescope at Lowell Observatory. The upper spectrum $(a)$ is from March 4 and shows relatively little $\mathrm{TiO}$ absorption, except for the band at $\lambda 4954$. Also present are numerous metallic absorption features. The structure in this spectrum is real and based upon weak $\mathrm{TiO}$ absorption and the width of the metal lines is classified as K3-4 III-I. The lower spectrum $(b)$ shown is from March 9 and shows a remarkable increase in TiO absorption bands from $(a)$, as well as a significant decrease in flux (the flux difference between the two spectra is real). It is classified as M3 III-I.

TABLE 1

PHOTOMETRIC EVOLUTION

\begin{tabular}{lccccc}
\hline \hline $\begin{array}{c}\text { Date of Observation } \\
\text { (UT) }\end{array}$ & $V$ & $U-B$ & $B-V$ & $V-R$ & $V-I$ \\
\hline 1994 Mar 3.67 & 8.67 & +1.22 & +1.65 & +0.93 & +1.82 \\
1994 Mar 4.67 & 8.76 & +1.4 & +1.73 & +0.96 & +1.91 \\
1994 Mar 7.71 & 9.82 & $\ldots$ & +1.97 & +1.07 & +2.27 \\
1994 Mar 8.70 & 10.29 & $\ldots$ & +1.94 & +1.20 & +2.59 \\
1994 Mar 10.71 & 11.18 & $\ldots$ & +1.85 & +1.32 & +3.09 \\
1994 Mar 11.4 & 12.56 & $\ldots$ & +1.63 & +2.01 & $\ldots$ \\
1994 Jun 5-6 & 18.78 & $\ldots$ & +0.90 & +4.12 & $\ldots$ \\
\hline
\end{tabular}

Note.-Photometry of Nova Sagittarii 94 by A. C. Gilmore with the $0.6 \mathrm{~m}$ $\mathrm{f} / 16$ Cassegrain at Mt. John Observatory (Wagner et al. 1994; Tomaney et al. 1994). Typical uncertainties are $0.05 \mathrm{mag}$ and 0.1 in $U-B(1 \sigma)$. The photometry listed for 1994 March 11 and June 5 was obtained by convolving the March 11 (Fig. 4) and June 5 (Fig. 5) spectra with standard filters. These measurements were then placed on an absolute scale by interpolating the photoelectric $V$ values shown in Fig. 2. We estimate the uncertainties to be approximately $10 \%$. 
TABLE 2

SpeCtroscopic ObSeRvations and Model Results

\begin{tabular}{|c|c|c|c|c|}
\hline $\begin{array}{l}\text { UT Date } \\
\text { (1) }\end{array}$ & $\begin{array}{l}\text { Spectral Coverage } \\
(\AA) \\
(2)\end{array}$ & $\begin{array}{l}\text { Resolution } \\
(\AA) \\
(3)\end{array}$ & $\begin{array}{l}\text { Telescope } \\
\text { (4) }\end{array}$ & $\begin{array}{l}\text { Model Temperature } \\
\text { (K) } \\
(5)\end{array}$ \\
\hline 1994 Mar $4.5 \ldots \ldots .$. & $4500-5700$ & 10 & $1.8 \mathrm{~m}$ Perkins & 4400 \\
\hline $1994 \operatorname{Mar} 7 . \ldots \ldots \ldots$ & $6200-6800$ & 1.4 & $3.5 \mathrm{~m} \mathrm{NTT}$ & $\ldots$ \\
\hline 1994 Mar $9.5 \ldots \ldots .$. & $4500-5700$ & 10 & $1.8 \mathrm{~m}$ Perkins & 3800 \\
\hline 1994 Mar $11 \ldots \ldots \ldots$ & $3800-8600$ & 7 & $3.6 \mathrm{~m} \mathrm{La}$ Silla & 3100 \\
\hline 1994 Mar $20.4 \ldots \ldots$. & $3750-9800$ & 13 & $3.6 \mathrm{~m}$ La Silla & 2600 \\
\hline 1994 Apr 26 ........ & $3670-9960$ & 0.5 & $1.5 \mathrm{~m}$ Palomar & $\ldots$ \\
\hline 1994 Jun $5 \ldots \ldots \ldots$. & $3650-9100$ & 3.5 & $4.5 \mathrm{~m} \mathrm{MMT}$ & 2300 \\
\hline
\end{tabular}

NoTE.-The log of our spectroscopic observations of V4332 Sgr. Col. (1) shows the date of observation, cols. (2) and (3) show the spectral range and resolution of the data, respectively, and col. (4) shows the telescope at which we obtained the data (see $\S 3$ ). Col. (5) shows the effective temperature we derived for each spectrum using the PHOENIX model atmosphere code (see $\S 5$ ).

In addition to the stellar absorption features, a narrow and unresolved $\mathrm{H} \beta$ emission line with an equivalent width of 3 $\AA$ is present. The March 9 spectrum (Fig. $3 b$ ), only 5 days later, shows the rapid spectral evolution of V4332 Sgr. During this interval, the spectral type changed from that of a K3-4 III-I star to that of a M3 III-I star, although atmospheric model fitting (see $\S 5$ below) yields an effective temperature consistent with a K5 III. This discrepancy is probably due to the limited wavelength range of this spectrum (see Table 2). Over this same 5 day period, the $V$-band brightness decreased by approximately $1 \mathrm{mag}$. Unresolved $\mathrm{H} \beta$ emission is present in this spectrum with an equivalent width of $11.4 \AA$. The TiO absorption features, which were weak or absent on March 4, are now conspicuous in the spectrum. Without more detailed spectra at higher spectral resolution in the blue or the near infrared, it is difficult to distinguish between luminosity classes of III and I at this spectral type. In any event, using luminosity classifications meant for static atmospheres on an outburst are likely to be very uncertain.

Beginning on March 11, we were able to secure spectra that covered a much larger portion of the visible wavelength region than our previous observations. In Figure $4 a$, we show the spectrum of V4332 Sgr obtained on 1994 March 11.4 with the $3.6 \mathrm{~m}$ telescope at La Silla using the ESO Faint Object Spectrograph and Camera (EFOSC). The spectrum covers the region $3800-8600 \AA$ at a resolution of $7 \AA$ and was obtained under nonphotometric conditions. Comparison with $\mathrm{M}$ star templates from the sources cited above suggests that the spectrum is that of a M5-6 III or M5-6 Ib-II star. At our resolution, it is difficult to distinguish between these luminosity classes. Emission lines arising from the Balmer series are now striking over our extended spectral range. The observed ratios of the $\mathrm{H} \alpha, \mathrm{H} \gamma$, and $\mathrm{H} \delta$ emission lines to $\mathrm{H} \beta$ are $2.6,0.8$, and 0.8 , respectively, with a formal measurement uncertainty of $10 \%$, although we note that there is significant additional uncertainty in estimating the true level of the underlying stellar continuum as well as absorption from the photosphere. In particular, our high-resolution spectra (see below) show absorption in the $\mathrm{H} \alpha$ profile, so clearly these line ratios are very approximate. These caveats apply to all of our reported line ratios.

On March 20.4, we obtained another spectrum with the $3.6 \mathrm{~m}$ telescope and EFOSC under photometric conditions at a resolution of $13 \AA$ and covering the range $3750-9800 \AA$
(Fig. 4b). This spectrum is more typical of a M6.5-7 III or II star since the VO bands at 7400 and $7900 \AA$ are now discernible. The observed ratios of the $\mathrm{H} \alpha, \mathrm{H} \gamma$, and $\mathrm{H} \delta$ emission lines to $\mathrm{H} \beta$ are $7.5,0.6$, and 0.7 , respectively, with a formal measurement uncertainty of $10 \%$. Weak emission due to $\mathrm{Mg}$ I at $\lambda 4571$ is also visible in both spectra, although no forbidden lines are apparent.

In Figure 5 we present a composite spectrum of V4332 Sgr obtained on 1994 June 5 and 6 using the $4.5 \mathrm{~m}$ Multiple Mirror Telescope (MMT) and the Blue Channel CCD spectrograph. The June 5 spectrum covers the region 3650$7200 \AA$, and the June 6 spectrum covers the region 5600 $9100 \AA$, both at a resolution of $3.5 \AA$. The spectrum continued to evolve strongly toward later spectral types and decrease overall in absolute flux from early March, although we cannot infer a $V$ magnitude from the spectrum because of slit losses. From the strength of the VO bands, we classify this spectrum as an M8-9 III. In addition to the narrow Balmer emission lines present in earlier spectra, emission lines arising from $\mathrm{Mg}$ I, $\mathrm{Na}$ I, [O I $], \mathrm{Fe}_{\mathrm{I}}$, and $\mathrm{Fe}$ II

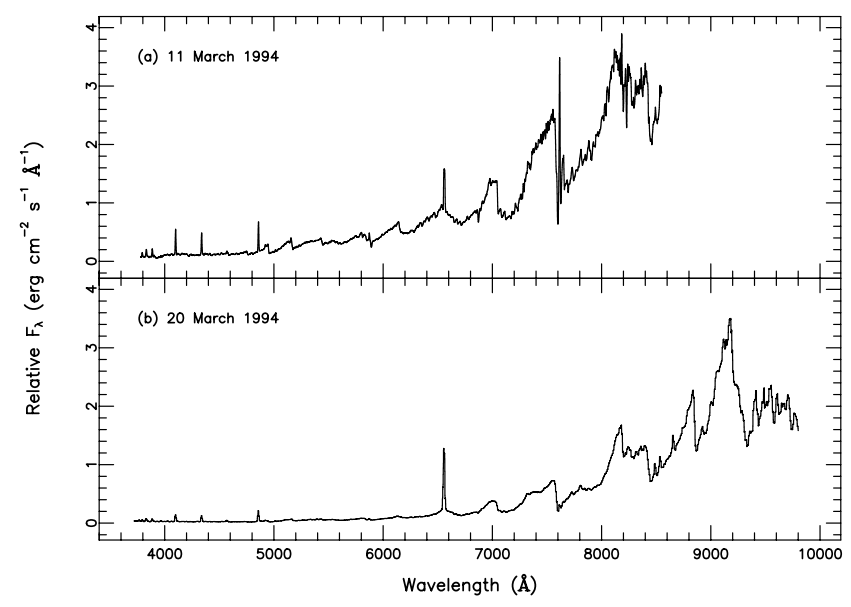

FIG. 4.-March 11 and 20 spectra obtained at the $3.6 \mathrm{~m}$ ESO telescope at La Silla. The upper spectrum $(a)$ is from March 11 and shows further evolution toward the red from March 9. The TiO bands have increased in strength, and the greater blue coverage of this spectrum shows the narrow Balmer lines in emission. We classify this spectrum as M5-6 III-II. The lower spectrum $(b)$ is from March 20 and shows weak VO bands at $\lambda \lambda 7400$ and 7900 characteristic of class M6.5. Again we find the luminosity class to be of type III-II. In addition to the narrow Balmer series present as in $(a)$, $\mathrm{Mg}_{\mathrm{I}} \lambda 4571$ is weakly present in emission in both spectra. 


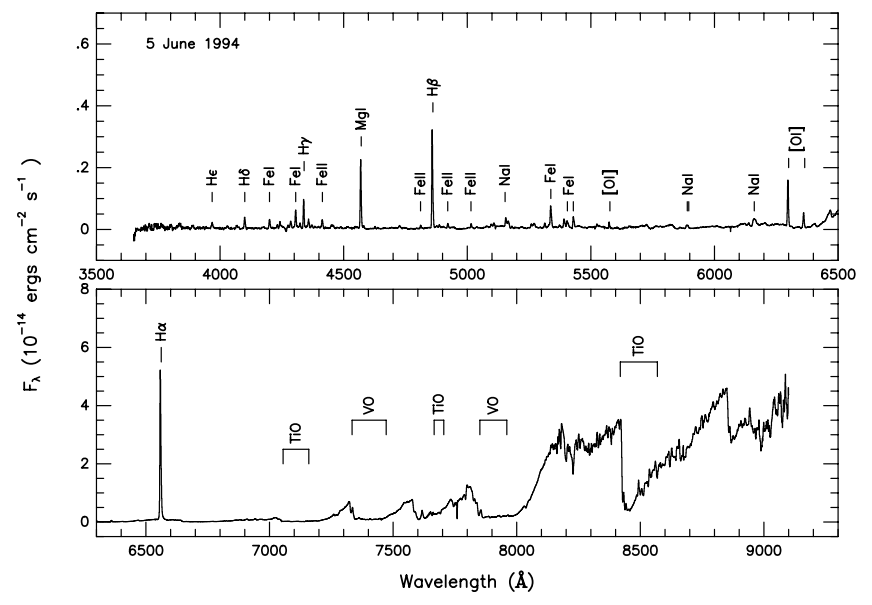

FIG. 5.- Spectrum taken on 1994 June 5-6 at the $4.5 \mathrm{~m}$ MMT. The bluer component of the spectrum is dominated by Balmer emission, $\mathrm{Mg} \mathrm{I}$, $\mathrm{Fe}$ I, Fe II, Na I, and [O I] (see also Table 3). The red component is dominated by strong absorption features, particularly $\mathrm{TiO}$ and $\mathrm{VO}$. We classify this spectrum as an M8-9 III.

are also present. The observed ratios of the $\mathrm{H} \alpha, \mathrm{H} \gamma$, and $\mathrm{H} \delta$ emission lines to $\mathrm{H} \beta$ are $18.6,0.26$, and 0.12 , respectively, again with a formal measurement uncertainty of $10 \%$. The equivalent widths of the Balmer series and other prominent lines are listed in Table 3.

Figure 6 shows the color excess evolution of V4332 Sgr for three dates on which we obtained spectra and also have relatively coincident color information. The points plotted in this figure represent the observed color excess of V4332 Sgr, which we derived from the difference between the published photoelectric photometry in Table 1 and the nominal color of giant stars (Johnson 1966) of our observed spectral type. The error bars represent an assumed uncertainty of 1 spectral type. This figure shows that the average $E(B-V)$ is relatively constant and consistent with the value we infer for March 4, $E(B-V) \sim 0.32 \pm 0.02$. If this component is due to interstellar reddening, then $A_{V} \simeq 1 \mathrm{mag}$

TABLE 3

LINE IDENTIFICATIONS FROM 1994 JUNE 5-6

\begin{tabular}{|c|c|c|}
\hline $\begin{array}{l}\text { Observed Wavelength } \\
\text { (§) } \\
(1)\end{array}$ & $\begin{array}{l}\text { Line Identification } \\
\text { (s) } \\
(2)\end{array}$ & $\begin{array}{c}\text { Equivalent Width } \\
(\AA) \\
(3)\end{array}$ \\
\hline $3889.3 \ldots$. & $\mathrm{Fe}$ I $\lambda 3887.1+\mathrm{H}_{8}$ & 62 \\
\hline $3968.1 \ldots \ldots \ldots \ldots \ldots \ldots$ & $\mathrm{H} \epsilon$ & 32 \\
\hline $4099.8 \ldots \ldots \ldots \ldots \ldots \ldots$ & $\mathrm{H} \delta$ & 115 \\
\hline $4200.3 \ldots \ldots \ldots \ldots \ldots \ldots$ & Fe I $\lambda 4202.0$ & 65 \\
\hline $4305.8 \ldots \ldots \ldots \ldots \ldots \ldots$ & Fe I $\lambda 4307.9$ & 30 \\
\hline $4338.3 \ldots \ldots \ldots \ldots \ldots \ldots$ & $\mathrm{H} \gamma$ & 102 \\
\hline $4413.5 \ldots \ldots \ldots \ldots \ldots \ldots$ & {$[\mathrm{Fe}$ II $] \lambda 4416.3$} & 36 \\
\hline $4569.0 \ldots \ldots \ldots \ldots \ldots \ldots$ & Mg I $\lambda 4571.1$ & 172 \\
\hline $4858.3 \ldots \ldots \ldots \ldots \ldots \ldots$ & $\mathrm{H} \beta$ & 305 \\
\hline $5337.9 \ldots \ldots \ldots \ldots \ldots$ & $\mathrm{Fe}$ I $\lambda 5341.0$ & 90 \\
\hline \multirow{2}{*}{$5889.7 \ldots \ldots \ldots \ldots \ldots \ldots$} & $\mathrm{Na}$ I $\lambda \lambda 5890.0$ & $\ldots$ \\
\hline & $+\mathrm{Na}$ I $\lambda 5895.9$ & 40 \\
\hline $6297.2 \ldots \ldots \ldots \ldots \ldots \ldots$ & {$[\mathrm{O}$ I] $\lambda 6300.2$} & 70 \\
\hline $6360.6 \ldots \ldots \ldots \ldots \ldots \ldots$ & {$[\mathrm{O} \mathrm{I}] \lambda 6363.9$} & 41 \\
\hline $6558.7 \ldots \ldots \ldots \ldots \ldots$ & $\mathrm{H} \alpha$ & 450 \\
\hline
\end{tabular}

Note.-The identifications of the brightest lines in the 1994 June 5-6 spectrum shown as Fig. 5. Col. (1) lists the observed wavelength of the emission feature, while col. (2) lists the identification of the line(s). The equivalent widths of the features are given in col. (3).

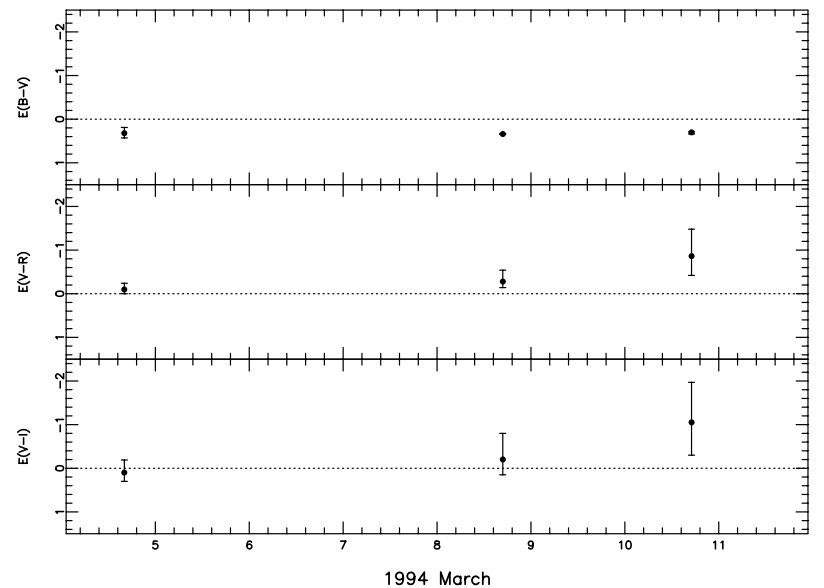

FIG. 6.-Excess color evolution of V4332 Sgr for the three dates on which we have both photometry and spectroscopy. We define the color excess as the difference between the observed color, based upon published photoelectric photometry, and the expected color of the object, based upon its temperature as derived from our spectra. The error bars shown correspond to the color difference for an assumed uncertainty of one spectral type. Photometric uncertainties are significantly less, particularly in the lower two frames. See $\S 4$ for further details.

(assuming $R=3.1)$. Both $E(V-R)$ and $E(V-I)$ appear to be somewhat bluer than the assumed colors for static atmospheres; this may be due to a poor match between the inferred temperatures and static atmosphere colors or scattering in the expanding envelope. The effect seen in Figure 6 is in the opposite sense of what one would expect if dust were forming in the envelope.

\section{MODELING OF THE SPECTRA}

We have modeled the spectra using the PHOENIX model atmosphere code (Hauschildt \& Baron 1998; Hauschildt, Allard, \& Baron 1999a). The presence of molecular bands in the observed spectra indicates low effective temperatures. Therefore, we have calculated models using essentially the same input physics as described in Hauschildt et al. (1999a). However, we have used spherical (rather than plane-parallel) geometry for the model construction because of the geometrical extension of the changing atmosphere. In addition, the models presented here were calculated using an improved water line list $(300$ million lines; Partridge \& Schwenke 1997), which replaces the older water data used in Hauschildt et al. (1999a). For this preliminary modeling we have neglected the small expansion velocity (see below) of the shell and used static atmosphere models in order to save computational time. The formation of the molecules and their lines were treated in LTE (molecular non-LTE models are in preparation but have not yet been finalized), and all models were calculated assuming solar abundances. Before the modeling, the observed spectra were corrected for interstellar reddening assuming $E(B-V)=0.32$ as derived above.

The largest difference between the spectra of V4332 Sgr and that of a "normal" classical nova atmosphere early in its outburst (the "iron curtain phase") is the significantly lower effective temperature. The emission lines that are apparent in the observed spectra are true emission features, evident by a comparison of the model spectra with the observed data. The models do not include a circumstellar shell where these lines probably form and thus cannot be 
expected to reproduce them. Our spectra do, however, resemble active M-type spectra (the gravities in the dMe's are larger, however) more than classical nova spectra. In a statistical sense, the model spectra do not reproduce the observed spectra very well. In part, this is due to the model simplifications (abundances and LTE). Another major problem is the incomplete line list data for $\mathrm{TiO}$ we had to use in these models (Hauschildt et al. 1999b) as well as unknown line data for molecules like $\mathrm{VO}$ and $\mathrm{FeH}$. However, we can say that the effective temperature of the spectra dropped throughout the observed time period by nearly a factor of 2 , while the spectra continued to stay optically thick. This is in sharp contrast to normal novae, where the effective temperature of the shell increases and becomes optically thin.

Our models fits to the March 4 and March 9 spectra show a decline in temperature from $\sim 4400$ to $3800 \mathrm{~K}$. While the March 4 fit is in good agreement with our spectral classification of K3 (see Fig. 7), our March 9 fit yields a hotter temperature than our spectral classification. This inconsistency is probably due to the narrow observed spectra range on this data. The model fits to March 11 $(\sim 3100 \mathrm{~K})$, March $20(\sim 2600 \mathrm{~K})$, and June 5-6 ( 2300 K) are all consistent with the spectral classification discussed above. Our derived effective temperatures for each of the spectra are listed in Table 2. In Figure 7 we show the
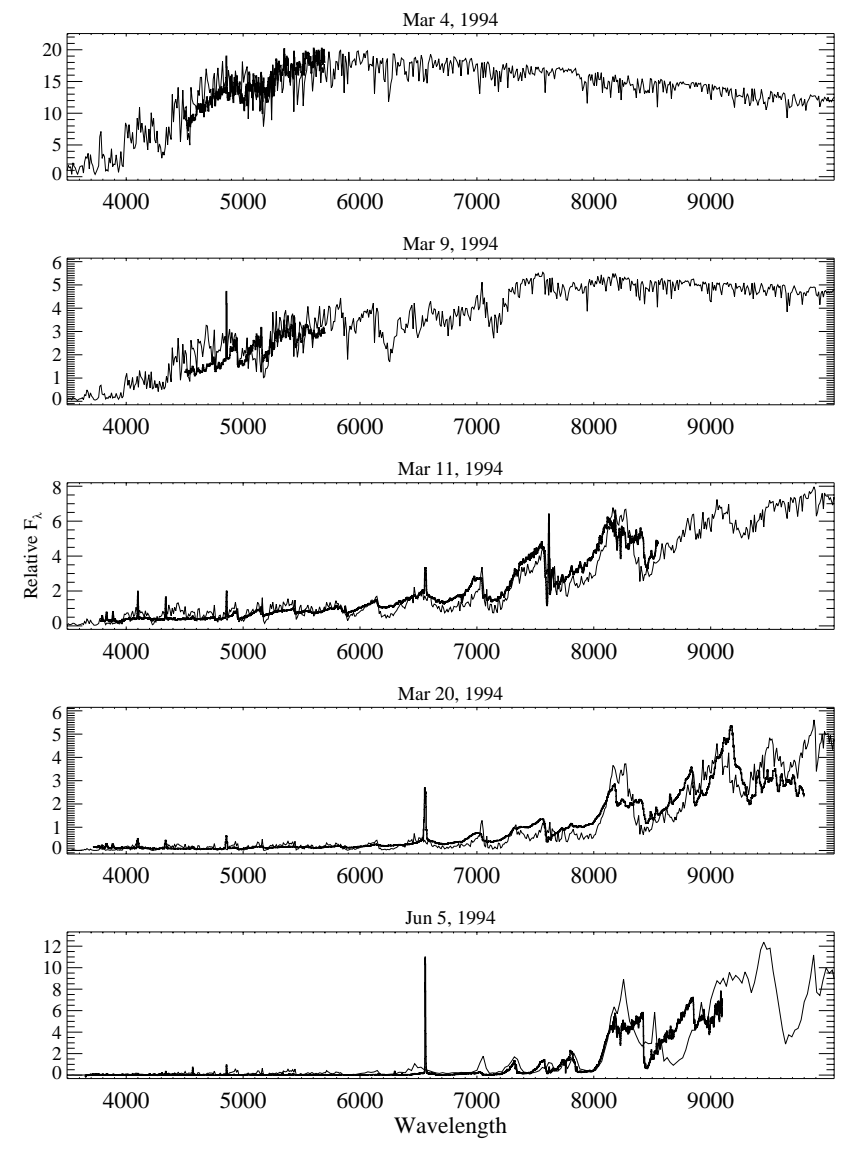

FIG. 7.-Comparison of the observed reddening corrected spectra of V4332 Sgr (thick lines) and the best-fit late-type stellar model atmosphere as described in the text (see $\S 5$ for details). A consistent spectral range of the model is shown to illustrate the fast spectral evolution of the V4332 Sgr. In particular, the effective temperatures of the model fits vary from $4400 \mathrm{~K}$ (March 4) to $2300 \mathrm{~K}$ (June 5-6). The temperatures corresponding to these best-fit models are listed in Table 2. observed spectra (thick lines) along with the model fits (thin lines).

The static atmosphere models we have used to obtain temperature estimates also provide us with a way to compare the change in apparent $V$ magnitude with the change in the bolometric luminosity. As shown on Table 1, V4332 Sgr decreases by approximately $10 \mathrm{mag}$ in $V$ over the 3 month period from the beginning of March to the beginning of June, over the same period when our model fits show a decrease in effective temperature from 4400 to 2300 $\mathrm{K}$. When we use the bolometric corrections of these models to calculate the change in the bolometric luminosity over this time, we find that the bolometric luminosity decreases by a factor of $\sim 100$ over this 3 month period.

\section{LINE PROFILES}

In order to learn more about the dynamics of the cool, evolving envelope indicated by our low-dispersion spectroscopy, we obtained a higher dispersion spectrum on 1994 March 7 in the $\mathrm{H} \alpha$ region using the $3.5 \mathrm{~m}$ New Technology Telescope (NTT) at La Silla with the ESO Multimode Instrument. Using a 1".1 wide entrance slit and a 1200 grooves $\mathrm{mm}^{-1}$ grism, we obtained a spectrum covering the range $6200-6800 \AA$ at a resolution of $1.4 \AA$. The region from 6545-6575 $\AA$ is shown in Figure 8a, in which the continuum normalized $\mathrm{H} \alpha$ profile is represented by crosses. The line profile consists of a narrow emission line blended with a

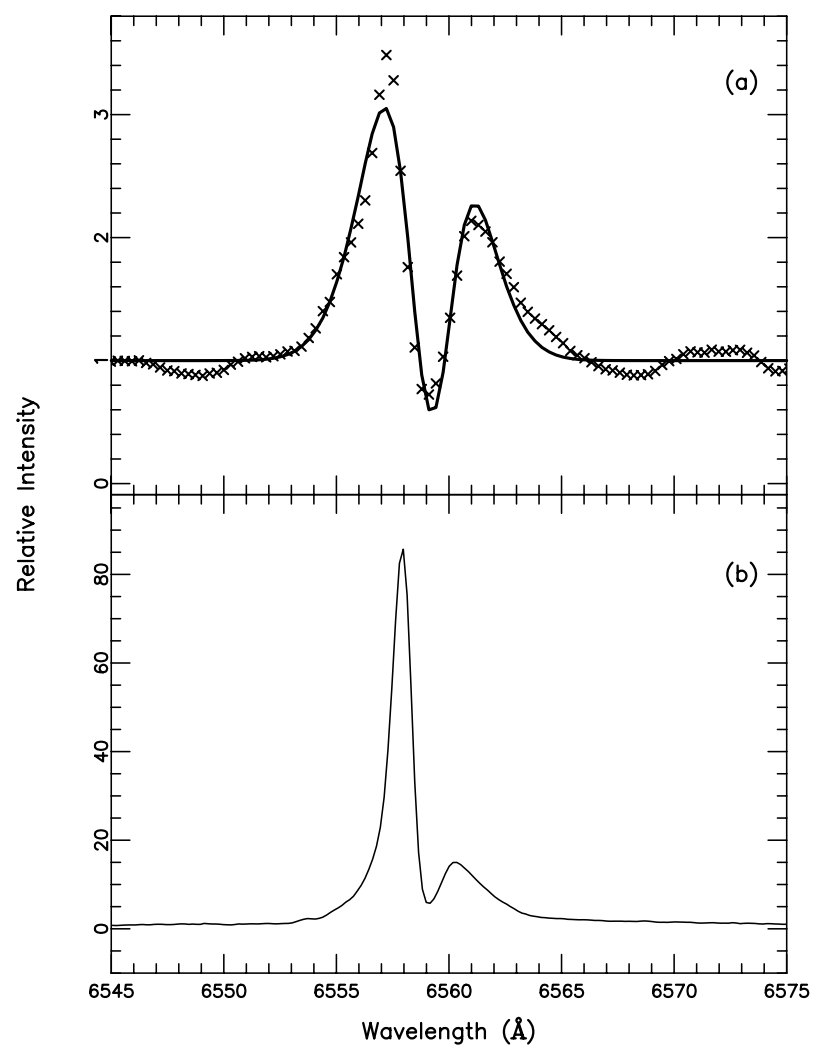

FIG. 8. - (a) High-resolution spectrum obtained 7 March 1994 at the ESO $3.5 \mathrm{~m}$ NTT. The spectrum, represented by crosses, is centered on the $\mathrm{H} \alpha$ line and has a resolution of $1.4 \AA$ pixel $^{-1}$. The line fit represents a simultaneous fit of two Gaussian components to the inverse P Cygni profile; the emission is centered approximately $20 \mathrm{~km} \mathrm{~s}^{-1}$ to the blue of the absorption feature. See $\S 6$ for further details. (b) $\mathrm{H} \alpha$ line profile obtained on 26 April 1994 at the Palomar $1.5 \mathrm{~m}$ telescope at a resolution of $1 \AA$. Note the dramatic change in the profile width between the two dates. 
redshifted but narrower absorption line, which together form an inverse P Cygni profile.

To extract quantitative information from this profile, we deconvolved the two components by Gaussian fitting with a nonlinear least-squares technique. Our best fit is shown as a solid line superposed on the observations in Figure 8a. We find that the absorption component is redshifted with respect to the emission component by $17 \mathrm{~km} \mathrm{~s}^{-1}$. The FWHM of $\mathrm{H} \alpha$ emission is $200 \mathrm{~km} \mathrm{~s}^{-1}$, while that of $\mathrm{H} \alpha$ absorption is $70 \mathrm{~km} \mathrm{~s}^{-1}$, both corrected for instrumental resolution. These results suggest that the $\mathrm{H} \alpha$ line-emitting region is expanding with a velocity of about $100 \mathrm{~km} \mathrm{~s}^{-1}$. If the emission and absorption components are moving with respect to the same center of mass, an infall velocity of about $20 \mathrm{~km} \mathrm{~s}^{-1}$ is required of a component of the envelope projected against the continuum source. Another striking aspect of Figure $8 a$ is that the systemic velocity of the mean $\mathrm{H} \alpha$ profile is blueshifted with respect to line center by 180 $\mathrm{km} \mathrm{s}^{-1}$. This blueshift is also evident in our other spectra (see, e.g., Table 3) and may be due to an occulting source in or near to the emission-line region, the effects of an opacity source in the envelope such as dust obscuring the far side of the emitting envelope, the actual heliocentric radial velocity of V4332 Sgr, or a combination of all of these effects.

On 1994 April 26 we obtained a second high-resolution spectrum of V4332 Sgr using the Palomar Observatory 1.5 $\mathrm{m}$ telescope and echelle spectrograph at a resolution of $1 \AA$. The spectrum covers the region from $3670-9960 \AA$ in 58 orders. We were unable to perform a sky subtraction or flux calibration for this spectrum since we lacked the appropriate calibration data. The emission lines we identified in the spectrum include the Balmer series of hydrogen and emission lines arising from $\mathrm{Ca}$ I $\lambda 4226, \mathrm{Ca}$ II $\mathrm{H}$ and $\mathrm{K}$ lines, the Ca II infrared triplet, $\mathrm{O}$ I $\lambda 8446$, weak [O I] $\lambda \lambda 6300$ and 6363, Na I D, Mn I multiplets 1 and 2 at $\lambda \lambda 5394,5432$, and 4030, and numerous emission lines from $\mathrm{Fe} \mathrm{I}$ and $\mathrm{Fe}$ II. The lack of sky subtraction makes positive identifications of other emission lines ambiguous. All of the identified emission lines exhibit the blueward shift described above. The [O I] detections are clearly not the telluric features since they are shifted blueward of the telluric lines. In Figure $7 b$, we show the $\mathrm{H} \alpha$ line profile obtained on April 26. The same basic structure is present in the line profile at this epoch as observed on March 7, except that the relative emissionand absorption-line strengths have changed and the line widths are narrower.

\section{COMPARISON WITH KNOWN OUTBURSTS AND VARIABLE STARS}

This object was referred to as a nova before the identification spectrum obtained by Wagner et al. (1994) showed that its spectrum was completely inconsistent with this classification. The collection of observations we present here show that this object also had a drastically different ejection velocity and temporal evolution from classical novae (Gallagher \& Starrfield 1978). Since novae do not fit the characteristics of this event, we investigated the possibility that this object could be another type of previously catalogued outburst. Symbiotic stars, for example, constitute a fairly diverse class of objects that exhibit a range of outburst phenomena (Kenyon 1986). The different classes of outburst exhibited by symbiotic stars, however, all include highionization emission lines and have much lower amplitudes than the observed $\gtrsim 10$ magnitude variation in V4332 Sgr.
Finally, the relatively rapid luminosity evolution of this object, the lack of evidence for periodicity, and the different spectral features make it unlikely that this object is an asymptotic giant branch star undergoing a final helium shell flash, such as FK Sgr.

\section{COMPARISON WITH M31 RV}

From the above comparison with known types of outbursts and variable stars, we find that V4332 Sgr does not conform to the criteria for membership in any of these classes. What is particularly striking is its unique spectral development from K3 III-I to M6 III-I in just 1 week and subsequently to M8-9 III in less than 3 months. Only the extremely luminous outburst in M31 in 1988, M31 RV (Rich et al. 1989; Mould et al. 1990), exhibited similar spectral evolution. Both M31 RV and V4332 Sgr were characterized by a rapidly evolving late-type stellar absorption-line spectrum and narrow Balmer emission lines with a rapidly increasing $\mathrm{H} \alpha-$ to- $\mathrm{H} \beta$ ratio with time. Both outbursts were not characteristic of classical novae, symbiotic stars, other known types of outbursts, or known variable stars, and neither have exhibited evidence of periodicity. In addition, the spectra of both objects exhibited no other emission lines than narrow Balmer emission lines until at least $t_{2}$ days after maximum.

M31 RV was discovered as a 15th magnitude red variable star 3' from the center of M31 on 1988 September 3 by Rich et al. (1989) during the course of a survey of luminous $M$ giants in the bulge of M31. It was independently discovered by Tomaney \& Shafter (1992) on 1988 August 18 during their study of novae in the bulge of M31 and by Bryan \& Royer (1992) on 1988 July 13 during their photographic survey for M31 novae at the Ford Observatory. The time of the outburst was constrained by Bryan \& Royer (1992) to have taken place sometime between 1988 June 14 and July 13 based on independent photographs of M31 obtained by A. N. Sollee.

Spectroscopy of M31 RV obtained by Rich et al. (1989) on 1988 September 5 exhibited unresolved $\mathrm{H} \alpha$ and $\mathrm{H} \beta$ emission, $\mathrm{Na}$ absorption, weak TiO bands, and a strong $\mathrm{Ca}$ II IR triplet. Based on the strength of the Ca II triplet, the lack of $\mathrm{CaH}$ absorption, and the strength of the $\lambda 6500$ blend (Ba II, $\mathrm{Ca} \mathrm{I}$, and $\mathrm{Fe} \mathrm{I}$ absorption blend), Rich et al. classified it as M0 Ie. Additional spectra (Mould et al. 1990) were obtained in early October and November 1988 and indicated that the spectrum of M31 RV had changed to later M types. By 1988 November 1, the spectrum was classified as M6 Ie. No emission lines other than the Balmer series were reported. Rich et al. reported dramatic variations in its radial velocity in their September 5 spectra in both the emission and absorption lines. Subsequent spectra obtained by Mould et al. (1990) gave a blueshift relative to the systemic velocity of M31 of $\simeq 150 \mathrm{~km} \mathrm{~s}^{-1}$.

The light curves of M31 RV and V4332 Sgr are very different in their relative rates of decline after maximum brightness. As measured by the time to decline 2 mag in brightness from maximum, the rate of decline for V4332 Sgr was $t_{2}=8$ days, whereas for M $31 \mathrm{RV}$ it was $t_{2}=78$ days. We note, however, that the time of maximum of both of these objects is somewhat uncertain. The maximum of M31 $\mathrm{RV}$ has been constrained by observation to only within a few weeks (Bryan \& Royer 1992; Sharov 1993). The time of maximum brightness of V4332 Sgr could be even more uncertain, since it could have reached maximum prior to its 
discovery. In either scenario, this would impact upon the value of $t_{2}$ used to characterize the temporal evolution of these objects.

Another difference between M31 RV and V4332 Sgr is the maximum absolute magnitude achieved during their outbursts. M31 RV reached an apparent magnitude of $\simeq 15$ in 1988 July. Assuming $(m-M)=24.2 \mathrm{mag}$ for M31, the bolometric absolute magnitude was $M_{\mathrm{bol}}=-10$ (Rich et al. 1989). While we do not know the distance to V4332 Sgr, there are many lines of evidence, such as the relatively weak interstellar absorption lines and low extinction estimate, which suggest that the progenitor was relatively nearby and similar to a late-type dwarf or giant star rather than a latetype supergiant. If we assume V4332 Sgr was characteristic of a $\mathrm{K}$ giant at maximum, as suggested by Figure 3, the implied distance is $\sim 300 \mathrm{pc}$, before including the effects of extinction. Performing the same exercise for subsequent points in the evolution leads to increasingly larger distance estimates as the object evolves toward later spectral types. The luminosity of this object is therefore not consistent with the observed spectral type and luminosity class, although as the luminosity increases with time over that expected for a star of the same spectral type, the distance estimate of $\sim 300$ pc derived from the first spectrum may be an upper limit. We note, as above, that this line of analysis is very uncertain because we do not expect the luminosity class of the object during outburst to provide a reasonable luminosity estimate in any event, since these luminosity classifications are based on static stellar atmospheres.

There is a spectroscopic similarity between V4332 Sgr and M31 RV in the strong increase in the $\mathrm{H} \alpha / \mathrm{H} \beta$ intensity ratio, possibly indicative of greatly increasing reddening at the source such as that produced by dust formation. V4332 Sgr had a $\mathrm{H} \alpha / \mathrm{H} \beta$ intensity ratio of 2.5 on March 11 $\left(t_{\text {dis }}+15\right.$ days, where $t_{\text {dis }}$ is the date of discovery, 1994 February 24). By June $5\left(t_{\mathrm{dis}}+40\right.$ days) this ratio had increased to $\sim 17$. Mould et al. (1990) reported M31 RV to have an $\mathrm{H} \alpha / \mathrm{H} \beta$ emission line ratio of 1.8 at $t_{\mathrm{dis}}+82$ days, while by $t_{\text {dis }}+111-119$ days, this ratio had increased to 9.8 (Tomaney \& Shafter 1992). Both Mould et al. (1990) and Tomaney \& Shafter (1992) interpret the continuum evolution and increase in the $\mathrm{H} \alpha / \mathrm{H} \beta$ emission-line ratio as due to dust formation. However, as we discuss below, we attribute this change to increasing optical depth in the hydrogen Balmer recombination lines.

\section{DISCUSSION}

Both M31 RV and V4332 Sgr do not conform to any known class of outburst. While M31 RV exhibited temporal and luminosity evolution orders of magnitude greater than V4332 Sgr, they may be related based upon their similar spectral evolution. M31 RV and V4332 Sgr evolved rapidly toward the red and exhibited an increasing $\mathrm{H} \alpha$-to- $\mathrm{H} \beta$ line emission ratio with time; thus it is possible that both outbursts produced a great deal of dust. However, the behavior of Figure 6 is inconsistent with this interpretation for V4332 Sgr. Except for a minor trend in the color excesses of $V-R$ and $V-I$ with time, which might be due to a systematic error with increasing spectral type in the intrinsic colors of $M$ giant stars, there is no indication that dust formation is taking place and resulting in large color excesses that would lead to large $\mathrm{H} \alpha / \mathrm{H} \beta$ intensity ratios. The observed colors are in good agreement with the temperatures derived from our atmosphere model without the addition of dust beyond our estimate of an interstellar extinction of $A_{V}=1$. Instead, large $\mathrm{H} \alpha / \mathrm{H} \beta$ intensity ratios can be produced if the Balmer lines become optically thick (case $\mathrm{C}$ recombination). This situation would then be similar to that observed in the evolution of the visible-wavelength spectra of type II supernovae. In their model for hydrogen recombination in SN 1987A, Xu et al. (1992) found large values for the $\mathrm{H} \alpha$-to-H $\beta$ ratio in their $N_{e}=10^{8} \mathrm{~cm}^{-3}, T=3000 \mathrm{~K}$ model for the late-time spectra.

We have independent evidence for such high densities and low temperatures in our spectra which further support these values for the physical parameters. As discussed in $\S 5$, our models show that the temperature changes from $T \sim 4400 \mathrm{~K}$ to $T \sim 2300 \mathrm{~K}$ over the 3 month period for which we have spectra. In addition, we detect [O I] $\lambda 5577$ in our 1994 June spectrum. The ratio of this line to the $\lambda 6300$ doublet is approximately 0.08 , implying $N_{e} \sim 10^{8}-10^{9}$ $\mathrm{cm}^{-3}$ (Begelman \& Sarazin 1986; Keenan et al. 1995). The ratio of the $\left[\mathrm{O}_{\mathrm{I}}\right] \lambda 6300$ doublet is $\sim 3$, however, indicating that the $\left[\mathrm{O}_{\mathrm{I}}\right]$ emission is optically thin. In addition, the absence of forbidden line emission from either outburst within $t_{2}$ of maximum suggests a high-density environment. The critical densities of bright, forbidden emission lines typically seen in outbursts are generally $10^{4}-10^{7} \mathrm{~cm}^{-3}$, and the absence of any of these lines provides a lower limit to the density of the line-emitting region.

Iben \& Tutkov (1992) suggested that M31 RV could be a nova-like outburst involving an uncharacteristically large amount of mass loss $\left(\sim 10^{-2} M_{\odot}\right)$. In their scenario, M31 $\mathrm{RV}$ could be the first outburst to occur in a short period $(80$ minutes to $2 \mathrm{hr}$ ) cataclysmic variable system, where the high mass loss and large luminosity would then be due to an exceptionally large mass build-up on a cold white dwarf. This large amount of mass loss could also explain the lack of a nebular phase because the large amount of ejected mass may be absorbing all of the ionizing photons from the central source. Our spectral and dynamical analysis of V4332 Sgr is not inconsistent with the Iben \& Tutkov (1992) model for M31 RV except for the lack of evidence for dust formation. However, further theoretical modeling is needed to see if such an outburst on a cold white dwarf could also produce the much lower maximum luminosity that V4332 Sgr exhibits. Such a great intrinsic variation in "first outburst " events could be explained, for instance, by highly variable mass loss and the proportion of energy that is emitted in the form of kinetic energy versus radiation.

An alternative explanation for this event and M31 RV could be a nuclear event in a single, evolved star that caused a slow shock to propagate through to the photosphere. This would push out the photosphere of the star, increasing the luminosity and correspondingly decreasing the effective temperature. This scenario explains the high densities, the low velocities, and the timescale we observe. This also provides a means of powering the event in the absence of any evidence for a UV source or that this object is in a binary system. Such evidence would consist of radial velocity variations, composite spectra, or evidence for a companion as the object faded, none of which have been observed. Our observations are also consistent with the ejection of a massive and opaque shell of material.

\section{SUMMARY}

We have presented photometry and spectroscopy that trace the evolution of V4332 Sgr, an unusual luminous red 
variable star in Sagittarius. Our data clearly rule out the possibility that this object was a nova or any other known type of variable object. In fact, the closest match to the spectral evolution of this object is $\mathrm{M} 31 \mathrm{RV}$, an extremely luminous and red variable that was observed in the bulge of M31 in 1988. However, significant differences exist between these two events. Particularly, the temporal evolution was much longer and the maximum luminosity much brighter for M31 RV. Therefore if these two events are similar, there must be substantial intrinsic variation in the outbursts.

Our model of the spectra observed over a 3 month baseline show that the effective temperature fell from $\sim 4400$ to $2300 \mathrm{~K}$. Our line diagnostic measurements in the later spectra, particularly the evolution of the $\mathrm{H} \alpha-$ to- $\mathrm{H} \beta$ ratio and the absence of strong forbidden lines, imply high densities $\left(N_{e}=10^{8}-10^{9} \mathrm{~cm}^{-3}\right)$ in addition to the low temperatures. Our observations of this object suggest a simple model in which a nuclear event in a single, evolved star caused a slow shock to drive the photosphere outward and resulted in the evolution to lower temperatures and the emission spectrum we see after three months. Further study of this object in quiescence as well as theoretical modeling based upon the observational data we present here will hopefully lead to a clearer understanding of the nature of this unusual outburst.

P. M. would like to thank Lowell Observatory for their hospitality while much of this work was completed. We would also like to acknowledge the support staff of Lowell Observatory, the NTT, La Silla, Palomar, and the MMT, where these observations were obtained. P. H. H. acknowledges partial support by NASA ATP grant NAG 5-3018, LTSA grant NAG 5-3619, and NSF grant AST-9720804 to the University of Georgia. Some of the calculations presented in this paper were performed on the IBM SP2 and SGI Origin 2000 of the UGA UCNS at the San Diego Supercomputer Center (SDSC) and at the National Center for Supercomputing Applications (NCSA), with support from the National Science Foundation, and at the NERSC with support from the Department of Energy. We thank all these institutions for a generous allocation of computer time. Thanks go to Phil Pinto, Anil Pradham, and Alison Sills for several helpful suggestions and Darren DePoy and the referee, Steve Shore, for many useful comments on the manuscript.
Begelman, M. C., \& Sarazin, C. L. 1986, ApJ, 302, L59

Bryan, J., \& Royer, R. E. 1992, PASP, 104, 179

Gallagher, J. S., \& Starrfield, S. 1978, ARA\&A, 16, 171

Gilmore, A. C., Kilmartin, P. M., Sakurai, I., \& Jones, A. F. A. 1994, IAU

Circ. 5943

Hauschildt, P. H., Allard, F., \& Baron, E. 1999a, ApJ, 512, 377

Hauschildt, P. H., et al. 1999b, in preparation

Hauschildt, P. H., \& Baron, E. 1998, J. Comput. Appl. Math., in press

Hayashi, S. S., Yamamoto, M., \& Hirosawa, K. 1994, IAU Circ. 5942

Iben, I., \& Tutkov, A. V. 1992, ApJ, 389, 369

Jacoby, G. H., Hunter, A. A., \& Christian, C. A. 1984, ApJS, 56, 257

Johnson, H. L. 1966, ARA\&A, 4, 193

Keenan, F. P., Aller, L. H., Hyung, S., \& Brown, P. J. F. 1995, PASP, 107, 148

Kenyon, S. J. 1986, The Symbiotic Stars (Cambridge: Cambridge Univ. Press)

Kilmartin, . 1994

Mould, J., et al. 1990, ApJ, 353, L35

\section{REFERENCES}

Partridge, H., \& Schwenke, D. W. 1997, J. Chem. Phys., 106, 4618

Pogge, R. W., Atwood, B., Byard, P. L., O'Brien, T. P., Peterson, B. M., Lame, N. J., \& Baldwin, J. A. 1995, PASP, 107, 1226

Rich, R. M., Mould, J., Picard, A., Frogel, J. A., \& Davies, R. 1989, ApJ, 341, L51

Sato, H., Sakurai, Y., Beers, T., Brandner, W., Lehmann, T., Duerbeck, H. W., Della Valle, M., \& Smette, A. 1994, IAU Circ. 6051

Sharov, A. S. 1990, Soviet Astron. Lett., 16, 85 .1993, Soviet Astron. Lett., 19, 33

Tomaney, A., Rich, R. M., Wagner, R. M., \& Della Valle, M. 1994, IAU Circ. 5949

Tomaney, A. B., \& Shafter, A. W. 1992, ApJS, 81, 683

Turnshek, D. E., Turnshek, D. A., Craine, E. R., \& Boeshaar, P. C. 1985 ,

An Atlas of Digital Spectra of Cool Stars (Tucson: Western Research)

Wagner, R. M. 1994, IAU Circ. 5944

Wenzel, W. 1994, IAU Circ. 5980

Xu, Y., McCray, R., Oliva, E., \& Randich, S. 1992, ApJ, 386, 181 